\title{
Grau de dependência nicotínica e valores espirométricos em acadêmicos tabagistas
}

\section{Degree of nicotine dependence and espirometric values in universitary smokers}

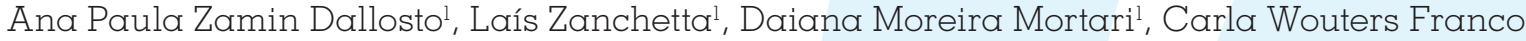 \\ Rockenbach2, Camila Pereira Leguisamo ${ }^{3}$ \\ ${ }^{1}$ Fisioterapeuta - UPF. Passo Fundo, RS - Brasil \\ ${ }^{2}$ Fisioterapeuta, especialista em Fisioterapia Cardiorrespiratória. Mestranda do curso de Medicina e Ciências da Saúde PUC/RS e \\ Professora no Curso de Fisioterapia - UPF. Passo Fundo, RS - Brasil \\ Fisioterapeuta, mestre e doutoranda em Ciências da Saúde- IC- FUC Porto Alegre, RS e Professora no Curso de Fisioterapia - UPF. \\ Passo Fundo, RS - Brasil.

\section{Endereço para correspondência} \\ Carla Wouters Franco Rockenbach \\ Silva Jardim, 68/402 - Centro \\ 99010-240 - Passo Fundo, RS [Brasil] \\ carlawfranco@upf.br
}

\begin{abstract}
Resumo
Objetivos: O estudo teve como objetivo verificar o grau de dependência nicotínica e valores espirométricos em acadêmicos tabagistas. Métodos: Foram selecionados acadêmicos tabagistas dos cursos de Fisioterapia e Psicologia da Universidade de Passo Fundo e avaliados o grau de dependência nicotínica; os valores espirométricos e o Pico de Fluxo Expiratório. A amostra foi composta por 32 indivíduos, sendo 24 do sexo feminino, com médias de idade e de tempo de tabagismo de 22,03 $\pm 2,68$ e $5,87 \pm 3,40$ anos, respectivamente. Resultados: Verificou-se que $87,5 \%$ dos indivíduos apresentavam um grau de dependência baixo; 9,3\%, moderada, e 3,1\%, alta. A média dos valores de $\mathrm{CVF}, \mathrm{VEF}_{1}$ e Índice de Tiffeneau ficaram acima do previsto, já a dos valores de pico de fluxo expiratório se mostrou reduzida, quando comparada aos valores previstos. Conclusão: A maioria dos jovens apresentou dependência nicotínica baixa e sintomas respiratórios leves apesar do pouco tempo de tabagismo, quanto aos valores espirométricos, obteve-se redução significativa no PFE.
\end{abstract}

Descritores: Espirometria; Dependência; Nicotina; Tabaco.

\begin{abstract}
Objectives: The study aimed to verify the nicotine dependence level and spirometric values in smoking academicians. Methods: The present study was performed with smoking students from the Psychology and Physiotherapy courses from the University of Passo Fundo, and evaluated the nicotine dependence, spirometric values and PEF (Peak Expiratory Flow). The sample was composed by 32 individuals, where 24 were female, with mean age 22,03 years ( $\pm 2,68$ years) and mean time of smoking 5.87 years ( $\pm 3,40$ years). Results: It was identified low nicotine dependence in $87,5 \%$ patients, $9,3 \%$ presented moderated dependence and 3,1\% high dependence. The mean values of FVC e FEV ${ }_{1}$ were higher than predicted, but the mean PEF presented reduced values compared to the predicted values. Conclusions: The most of young people presented low nicotine dependence and light respiratory symptoms, despite of the short time of smoking. On the espirometric values was observed a significant reduction in the PEF.
\end{abstract}

Key words: Espirometry; Dependence; Nicotine; Tobacco. 


\section{Introdução}

No Brasil, onde um terço da população adulta é fumante, estudos recentes apontam um aumento da prevalência de tabagismo em grupos específicos, como mulheres e jovens ${ }^{1}$.

Segundo a Organização Mundial de Saúde (OMS), o tabagismo é, atualmente, causa de 4,9 milhões de mortes por ano, no mundo, sendo previsto que esse número aumente para 7 milhões até o fim de 2020.

A principal substância do tabaco é a nicotina, droga que apresenta alto poder de modificar a biologia e a fisiologia do cérebro, sendo fortemente indutora de dependência. $\mathrm{O}$ conhecimento dos efeitos deletérios do hábito de fumar revela que o tabaco é a causa mais comum de morte evitável ${ }^{2}$.

Nesta última década, muitos são os estudos de prevalência de tabagismo, em que se verifica uma diminuição de tabagismo em adultos e aumento no consumo entre os jovens ${ }^{3}$.

O hábito de fumar se instala precocemente, já que $80 \%$ dos atuais adultos fumantes declaram tê-lo iniciado antes dos 18 anos de idade ${ }^{4}$.

O uso do tabaco durante a infância e a adolescência causa problemas de saúde significativos nos jovens, que apresentam sintomas, tais como tosse e expectoração, aumento do número e da gravidade dos problemas respiratórios e decréscimo do rendimento físico. Iniciar a fumar cedo pode provocar, em idade cada vez mais precoce, o aparecimento dos problemas de saúde relacionados ao consumo de tabaco, como câncer de pulmão e infartos do miocárdio ${ }^{5}$.

$\mathrm{O}$ entendimento de que o tabagismo deve ser prevenido e controlado na juventude, motivou a realização desta pesquisa. Com esses elementos, pretende-se contribuir para a reflexão e proposição de estratégias para prevenir o tabagismo entre os estudantes. Os profissionais de saúde assumem um papel de importância vital frente à prevenção do uso de tabaco; assim sendo a parceria entre a educação e a saúde pode promover o desenvolvimento de uma consciência crítica, favorecendo a adoção de hábitos e atitudes saudáveis ${ }^{6}$.
Neste contexto, objetivou-se verificar o grau de dependência nicotínica e valores espirométricos em acadêmicos tabagistas, em cursos de nível superior da área da saúde.

\section{Materiais e métodos}

Realizou-se um estudo caráter transversal, cuja amostra foi composta por acadêmicos dos cursos de Fisioterapia e Psicologia da Universidade de Passo Fundo (RS), selecionados aleatoriamente. Este trabalho foi aprovado pelo Comitê de Ética em Pesquisa da Universidade de Passo Fundo, protocolo 301/2007.

Após consentimento da coordenação dos cursos, foi exposto aos acadêmicos a importância e os objetivos da pesquisa, para a posterior assinatura do Termo de Consentimento Livre e Esclarecido (TCLE), de acordo com as normas da resolução 196/96 sobre pesquisa com seres humanos. Os sujeitos participantes preencheram uma ficha de avaliação constando dados pessoais (nome, idade, peso, altura) e um questionário sobre as particularidades do hábito tabágico, tais como anos de tabagismo; presença de amigos ou familiares tabagistas; aprovação familiar do hábito; presença de algum sintoma respiratório (tosse seca, dispneia, chiado no peito, expectoração); consciência dos males do hábito tabágico; se o sujeito se considera viciado; se houve tentativa ou vontade de cessação do hábito e, por fim, quais as razões que o levaram a fumar. Nessa última questão, poderia ser obtida mais de uma resposta (diminui a ansiedade, amigos fumam, para não engordar, sente prazer em fumar, estar aborrecido).

Foi também realizado o teste de Fagerström para identificar a dependência nicotínica, além da microespirometria e da mensuração do Pico de Fluxo Expiratório.

O teste de Fagerström é constituído de cinco questões, sendo que para cada pergunta há um valor correspondente. Os valores, quando somados, resultam em um escore que indica o grau de dependência nicotínica: baixa ( 0 a 4 pontos), moderada (5 pontos) ou alta (5 a 10 pontos) ${ }^{7}$. 
A avaliação da função pulmonar foi realizada por meio da microespirometria, objetivando verificar os valores de CVF, $\mathrm{VEF}_{1}$ e Índice de Tiffeneau dos universitários, com a utilização do microespirômetro da marca Micro Medicalâ. Cabe ressaltar que as manobras e os valores previstos estavam de acordo com as orientações da Sociedade Brasileira de Pneumologia e Tisiologia para as Provas de Função Pulmonar, encontradas no I Consenso Brasileiro Sobre Espirometria ${ }^{8}$.

O teste de Pico de Fluxo Expiratório foi realizado em seguida, utilizando-se o aparelho Assessâ que mensura o fluxo expiratório máximo do paciente em L/mim. Para efetuar as manobras e colher os valores previstos seguiram-se as orientações da Sociedade Brasileira de Pneumologia e Tisiologia para as Provas de Função Pulmonar.

As estatísticas descritivas foram computadas considerando-se a frequência e a média, e as exploratórias, por intermédio de figuras e tabelas. Para análises categóricas foram utilizados cálculos de média e DP (desvio-padrão), e para variáveis contínuas, utilizou-se o teste " $\mathrm{t}$ " Student, sendo considerado nível de significância quando $\mathrm{p}<0,001$. Usou-se para essas análises o pacote estatístico SPSS 10.0 e Windows Microsoft Excel.

\section{Resultados e discussão}

A amostra foi composta por 32 jovens universitários tabagistas, sendo 24 do sexo feminino (75\%), e 8 , do masculino ( $25 \%$ ), com média de idade de 22,03 $\pm 2,68$ anos, e tempo médio de 5,87 $\pm 3,40$ anos de hábito tabágico. No que se refere ao teste de dependência nicotínica, 87,5\% (28) dos indivíduos mostraram dependência baixa; 9,3\% (3), moderada, e 3,1\% (1) alta. Dos indivíduos avaliados, $62,5 \%$ (20) relataram que apresentam algum tipo de sintoma respiratório como tosse, dispneia, expectoração (Tabela 1).

O tabaco provoca dependência de modo progressivo, crônico e recorrente, sendo essa determinada por fatores farmacológicos, o que explica o fato de a maioria dos jovens apresentarem um número baixo na pontuação do teste de Fagerström em um estudo realizado para avaliar os conhecimentos, hábitos e grau de dependência de jovens fumantes ${ }^{6}$, o que vem ao encontro dos achados deste estudo.

Tabelal: Caracterização da amostra

\begin{tabular}{c|l}
\hline Sexo feminino & 24 \\
Sexo masculino & 8 \\
Idade (anos) & $22,03 \pm 2,68$ \\
Tabagismo (anos) & $5,88 \pm 3,40$ \\
Teste de Fagerström & \\
Dependência baixa & $87,5 \%$ \\
\hline Dependência moderada & $9,3 \%$ \\
Dependência alta & $3,1 \%$ \\
Presença de sintomas respiratórios & $62,5 \%$ \\
\hline
\end{tabular}

Em um trabalho realizado por Miedinger', observou-se, assim como nesta pesquisa, baixa pontuação para dependência nicotínica, quando analisados tabagistas jovens, resultado esse que favorece uma possível intervenção para cessação tabágica.

É possível observar que a amostra deste trabalho não apresentou alteração da função pulmonar, quando avaliado $\mathrm{VEF}_{1}, \mathrm{CVF}$ e índice de Tiffenau, cujos valores ficaram acima do previsto (Tabela 2).

Já em um estudo realizado para avaliar as alterações da função pulmonar causadas pelo tabagismo em adultos jovens, verificou-se pequena diminuição em $V F_{1}$ e CVF, nessa amostra ${ }^{10}$. Em outro trabalho avaliando a função pulmonar em

Tabela 2: Valores da espirometria

\begin{tabular}{|c|c|c|c|}
\hline Variável & Média & Test " $t$ " & Significância \\
\hline CVF & 3,339 & \multirow{2}{*}{2,223} & \multirow{2}{*}{$0,027585281^{*}$} \\
\hline CVF previstos & 2,029 & & \\
\hline $\mathrm{VEF}_{1}$ & 3,233 & \multirow{2}{*}{5,3261} & \multirow{2}{*}{$>0,000^{*}$} \\
\hline $\mathrm{VEF}_{1}$ previstos & 2,611 & & \\
\hline $\begin{array}{l}\text { Índice de } \\
\text { Tiffeneau } \\
\left.\text { (VEF }_{1} / C V F\right)\end{array}$ & 11,48245383 & \multirow[t]{2}{*}{10,241} & \multirow[t]{2}{*}{$>0,000^{*}$} \\
\hline $\begin{array}{c}\text { Índice de } \\
\text { Tiffeneau (VEF } \\
\text { /CVF) previstos }\end{array}$ & 11,40065 & & \\
\hline
\end{tabular}


adolescentes tabagistas, identificou-se significativa diminuição do $\mathrm{VEF}_{1}, \mathrm{CVF}$ e no $\mathrm{PFE}^{3}$.

Quando avaliado o número de cigarros fumados por dia, verificou-se que indivíduos que fumam mais de 20 unidades apresentam maior deterioração do $\mathrm{VEF}_{1}$, independentemente da duração do hábito de fumar ${ }^{11}$. Diante disso, estudos apontam que a obtenção de testes espirométricos e a apresentação desses resultados para os indivíduos fumantes têm sido defendidas como ferramentas motivacionais, que incentivam a cessação tabágica, fazendo com que os jovens abandonem esse hábito, antes que lhes tragam problemas pulmonares ${ }^{12}$.

Os valores de PFE, ilustrados na Tabela 3, demonstraram uma redução significativa $(\mathrm{p}<0,001)$, quando comparados com valores previstos de acordo com a idade e a altura. Os resultados vão ao encontro do estudo realizado por Fernandes et al. ${ }^{13}$, em que se verificou redução dos valores de PFE nos universitários tabagistas.

O tabagismo já tem seus efeitos comprovados sobre a redução do pico de fluxo em jovens que fumam ou moram em casas nas quais há algum familiar com hábito tabágico ${ }^{14}$. Em contrapartida, em outra pesquisa, em que também se mensurou o pico de fluxo expiratório, observou-se má reprodutibilidade dos valores de PFE entre indivíduos fumantes e os com tosse e catarro ${ }^{15}$.

\begin{tabular}{|c|c|c|c|}
\hline Variável & Média & Test " $\mathrm{t}$ " & Significância \\
\hline Peak Flow & 414,06 & 3,371 & $0,001^{*}$ \\
\hline $\begin{array}{c}\text { Peak Flow } \\
\text { previsto }\end{array}$ & 429,56 & & \\
\hline
\end{tabular}

Neste estudo, todos os indivíduos afirmaram ter amigos ou familiares fumantes. Quando questionados sobre a aprovação do hábito tabágico pelos familiares, 93\% (29) dos indivíduos referiram não ter aprovação. Todos os avaliados demonstraram ter consciência dos males do cigarro, e desses $65,6 \%$ (21) se consideram viciados no hábito tabágico; somente $18,8 \%$ desses indivíduos referiram terem feito uma tentativa para cessação
Tabela 4: Peculiaridades do hóbito tabágico

\begin{tabular}{c|c|c}
\hline Variável & Sim & Não \\
\hline $\begin{array}{c}\text { Aprovação do hábito pelos } \\
\text { familiares }\end{array}$ & 2 & 30 \\
\hline $\begin{array}{c}\text { Tem consciência sobre os males } \\
\text { do cigarro }\end{array}$ & 32 & 0 \\
\hline Considera-se viciado & 21 & 11 \\
\hline Tentativas para parar de fumar & 26 & 6 \\
\hline Quer parar de fumar & 24 & 8 \\
\hline
\end{tabular}

do tabagismo e $25,5 \%$ relataram não ter interesse em parar de fumar. Os dados obtidos podem ser visualizados na Tabela 4.

Verificou-se, neste estudo, uma forte associação entre o ato dos estudantes de experimentar o fumo ou de se tornarem fumantes com a presença de irmãos e amigos tabagistas, o que confirma os achados de Pinto e Ribeiro ${ }^{16}$. Esses autores relataram que os grupos de fumantes adolescentes exercem grande influência sobre jovens de mesma faixa etária nas fases iniciais de uso do tabaco, uma vez que as primeiras tentativas de fumar ocorrem entre irmãos e amigos, o que pode gerar expectativas, sugestões e reforço na manutenção do hábito.

Uma explicação para a iniciação precoce do hábito tabágico é o fato de crianças e adolescentes acreditarem tratar-se de um comportamento de adultos, repetindo-o na tentativa de assemelharse a eles. Crianças e adolescentes de pais fumantes têm maior tendência a fumar que filhos de não fumantes. Portanto, o grupo social em que os jovens estão inseridos parece contribuir para a adoção desse hábito ${ }^{6}$.

A Figura 1 mostra as razões que levam os estudantes a fumar. Observou-se que 30\% indicaram o cigarro como inibidor da ansiedade, 27,5\% afirmaram que sentem prazer em fumar, 18,29\% fumam, pois os amigos também o fazem, e 18,7\%, quando se sentem aborrecidos. Apenas $7 \%$ dos indivíduos afirmaram que fumam para não engordar.

Já Langhammer et. al. ${ }^{17}$ constataram que para as mulheres, quando comparadas com os homens, é mais difícil a cessação do consumo de tabaco, uma vez que ele beneficia o controle de peso. 


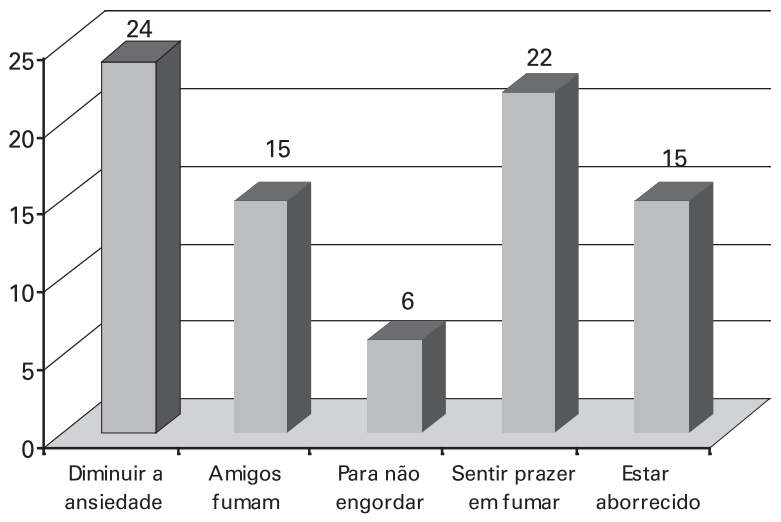

Figura l: Razões que levam o indivíduo a fumar

Essa preferência dos jovens pelo consumo tabágico é preocupante e representa a realidade atual entre os jovens.

Vale ressaltar que a idade de experimentação e a de início do hábito tabágico precoce, ou seja, antes dos 20 anos, estão comumente associadas ao período de transição do indivíduo do ensino médio para o superior. Muitos jovens podem ter o primeiro contato com o cigarro quando estão ingressando na universidade; portanto, é imprescindível tomar medidas antitabágicas para evitar que esse hábito se inicie na escola ${ }^{4}$.

Um estudo relata que apesar de haver grande número de estudantes tabagistas na área da saúde, eles fumam menos que a população em geral, talvez por terem consciência de suas responsabilidades, já que têm uma função importante na prevenção e erradicação do tabagismo na comunidade ${ }^{18}$.

Ao analisar a evolução da epidemia tabágica em adolescentes portugueses escolarizados, verificou-se um aumento no consumo tabágico entre voluntários do sexo feminino, quando comparados ao do masculino, sobretudo nas faixas etárias mais elevadas ${ }^{5}$.

Sugere-se a realização de estudos que comportem uma amostra maior e abranjam todos os cursos da área da saúde para se ter uma visão mais ampla da dimensão do problema. Dando-se a devida atenção a esse assunto, podem-se formular estratégias de atuação, principalmente na prevenção do tabagismo, fazendo com que os dados alarmantes de aumento no número de tabagistas sejam, enfim, revertidos.

\section{Conclusão}

A maioria dos jovens avaliados apresentou grau de dependência nicotínica baixa.

Constatou-se que valores espirométricos como $\mathrm{CVF}, \mathrm{VEF}_{1}$ e Índice de Tiffeneau não obtiveram alteração, quando relacionados aos previstos. Já o PFE, apresentou-se significantemente abaixo do valor previsto nos indivíduos analisados. Apesar do número importante de anos de tabagismo para a média de idade, os jovens mostraram sintomas respiratórios leves, como tosse seca, dispneia e pequena quantidade de expectoração.

Observou-se ainda que adolescentes que fumam há mais tempo tem consciência dos males do cigarro à saúde, porém não demonstraram interesse em abandonar o hábito tabágico, mesmo sem aprovação familiar.

O período universitário pode oferecer muitas oportunidades de intervenção no hábito tabágico dos alunos fumantes. O estabelecimento de medidas que auxiliem a cessação do tabagismo contribui, ao longo do tempo, para a diminuição dos valores de prevalência de jovens fumantes.

\section{Referências}

1 Chatkin JM. A influência da genética na dependência tabágica e o papel da farmacogenética no tratamento do tabagismo. J Bras Pneumol. 2006;32(6):573-9.

2 Araújo AJ, Menezes AMB, Dórea AJPS, Torres BS, Viegas CAA, Silva CAR, et al. Diretrizes para cessação do tabagismo. J Bras Pneumol. 2004;30(Suppl 2):S1-S76.

3 Vergara SL, Fernández CG, Hernández CO, Martínez ED, Delgado JMD, Suárez M D. Función pulmonar y exposición al humo del tabaco en adolescentes. An Pediatr (Barc) 2007;67(6):559-66. 
4 Andrade APA, Viegas CAA. Prevalência e característica do tabagismo em jovens da Universidade de Brasília. J Bras Pneumol. 2006;32(1):23-8.

5 Macedo M, Precioso J. Evolução da epidemia tabágica em adolescentes portugueses escolarizados e vias para o seu controle - uma análise baseada nos dados do Health behaviour in school- aged children. Rev Port Pneumol. 2006;12(5):525-38.

6 Almeida AF, Mussi FC. Tabagismo: conhecimentos, atitudes, hábitos e grau de dependência de jovens fumantes em Salvador. Rev Esc Enferm. USP. 2006;40(4):456-63.

7 Fagerström KO. Measuring degree of physical dependence to tobacco smoking with reference to individualization of treatment. Addict Behav. 1978;3(3-4):235-41.

8 Pereira CAC, Lemle A, Algranti EI. I Consenso brasileiro sobre espirometria. J Pneumol. 1996; 22(3):150-6.

9 Miedinger D, Chhajed PN, Karli C, Lupi C, Leuppi JD. Respiratory symptoms and smoking behaviour in Swiss conscripts. Swiss Med Wkly. 2006;136(4142):659-63.

10 Amigo H, Oyarzun MG, Bustos P, Rona RJ. Respiratory consequences of light and moderate smoking in young adults in Chile. Int J Tuberc Lung Dis. 2006;10(7):744-9.

11 Urrutia I, Capelastegui A, Quintana JM, Muniozguren N, Basagana X, Sunyer J. Smoking habit, respiratory symptoms and lung function in young adults. Eur J Public Health. 2005;15(2):160-5.
12 Wilt T, Niewoehner D, Kane R, Macdonald R, Joseph A. Spirometry as a motivational tool to improve smoking cessation rates: a systematic review of the literature. Nicotine Tob Res. 2007; 9:21-32.

13 Fernanes JR, Hassegawa, Macedo F, Montenegro S, Rangel RM, Redondo Kl, et al.

14 Análise comparativa do peak flow em indivíduos tabagistas e não tabagistas. [acesso em 2008 out. 20]. Disponível em: http://www.kathialr.hpg.com.br/ saude/10/index_int_12.html

15 Helden SNV, Helden EGV, Helden PDV. Factors influencing peak expiratory flow in teenage boys. $S$ Afr Med J. 2001;91(11):996-1000.

16 Holcroft C, Eisen E, Sama S, Wegman D. Measurement characteristics of peak expiratory flow. Chest. 2003;124(2):501-10.

17 Pinto D da S. Variáveis relacionadas à iniciação do tabagismo entre estudantes do ensino médio de escola pública e particular na cidade de Belém-PA. J Bras Pneumol 2007;33(5):558-64.

18 Langhammer A, Johnsen R, Holmen J, Gulsvik A, Bjermer L. Cigarette smoking gives more respiratory symptoms among women than among men. J Epidemiol Community Health. 2000;54:917-22.

19 Menezes A, Hallal P, Silva F, Souza M, Paiva L, D'ávila A, Weber B, Vaz V, Marques F, Horta B. Tabagismo em estudantes de Medicina: tendências temporais e fatores associados. J Bras Pneumol. 2004;30(3):223-8. 